\title{
Trajectory Planning and Tracking for Autonomous Overtaking: State-of-the-Art and Future Prospects
}

\author{
Shilp Dixit ${ }^{\mathrm{a}, *}$, Saber Fallah ${ }^{\mathrm{a}}$, Umberto Montanaro ${ }^{\mathrm{a}}$, Mehrdad Dianati ${ }^{\mathrm{b}}$, Alan \\ Stevens ${ }^{\mathrm{c}}$, Francis Mccullough ${ }^{\mathrm{d}}$, Alexandros Mouzakitis ${ }^{\mathrm{d}}$ \\ ${ }^{a}$ Department of Mechanical Engineering Sciences, University of Surrey, Guildford, Surrey, \\ GU2 7XH, United Kingdom \\ ${ }^{b} W M G$, International Manufacturing Centre, University of Warwick, Coventry, CV4 7AL, \\ United Kingdom \\ ${ }^{c}$ TRL, Wokingham, Berks, RG40 3GA, United Kingdom \\ ${ }^{d}$ Jaguar Land Rover Limited, Coventry, CV3 4LF, United Kingdom
}

\begin{abstract}
Trajectory planning and trajectory tracking constitute two important functions of an autonomous overtaking system and a variety of strategies have been proposed in the literature for both functionalities. However, uncertainties in environment perception using the current generation of sensors has resulted in most proposed methods being applicable only during low-speed overtaking. In this paper, trajectory planning and trajectory tracking approaches for autonomous overtaking systems are reviewed. The trajectory planning techniques are compared based on aspects such as real-time implementation, computational requirements, and feasibility in real-world scenarios. This review shows that two important aspects of trajectory planning for high-speed overtaking are: (i) inclusion of vehicle dynamics and environmental constraints and (ii) accurate knowledge of the environment and surrounding obstacles. The review of trajectory tracking controllers for high-speed driving is based on different categories of control algorithms where their respective advantages and disadvantages are analysed. This study shows that while advanced control methods improve track-
\end{abstract}

\footnotetext{
*Corresponding author

Email addresses: s.dixit@surrey.ac.uk (Shilp Dixit), s.fallah@surrey.ac.uk (Saber Fallah), u.montanaro@surrey.ac.uk (Umberto Montanaro), m.dianati@warwick.ac.uk (Mehrdad Dianati), astevens@trl.co.uk (Alan Stevens), fmccull2@jaguarlandrover.com (Francis Mccullough), amouzak1@jaguarlandrover.com (Alexandros Mouzakitis)
} 
ing performance, in most cases the results are valid only within well-regulated conditions. Therefore, existing autonomous overtaking solutions assume precise knowledge of surrounding environment which is not representative of real-world driving. The paper also discusses how in a connected driving environment, vehicles can access additional information that can expand their perception. Hence, the potential of cooperative information sharing for aiding autonomous high-speed overtaking manoeuvre is identified as a possible solution.

Keywords: autonomous vehicles, overtaking, trajectory planning, trajectory tracking, connected vehicles

\section{Introduction}

Modern cars are equipped with various sensors and electronic systems to reduce the workload of a driver by providing emergency assistance (e.g., ABS, traction control, stability control, etc.), ADAS (e.g., cruise control, lane keep-

5 ing, crosswind assistance, blind spot detection, etc.), and navigational assistance (e.g., trip planning, route selection, regular traffic update, etc.). However, the next generation of intelligent vehicles are expected to have increased capabilities which allow automated manoeuvring in various driving scenarios [1, 2]. Overtaking is one of the most common driving manoeuvre and any vehicle capable of end-to-end autonomy must have the ability to determine if, when, and how to perform this driving task.

Overtaking is a complex driving task as it involves both lateral and longitudinal motions of an overtaking vehicle (subject vehicle) while avoiding collisions with a slower moving vehicle (lead vehicle) 3 . Additional complexity arises due to different environmental conditions (e.g., road legislations, visibility, weather, etc.) and diversity of road-users (e.g., small cars, buses, trucks, etc.) 4]. Typically, an overtaking manoeuvre is considered successful on proper completion of three sub-manoeuvres namely, (i) lane change to overtaking lane, (ii) pass lead vehicle(s), and (iii) lane change back to original lane [5]. The lane change sub-manoeuvre which indicates the start and the end of an overtake can be 
classified under two categories; (i) Discretionary Lane Change (DLC) and (ii) Mandatory Lane Change (MLC) [6]. A DLC sub-manoeuvre is performed when the immediate traffic situation in the faster lane is deemed to be better than the current lane and thus, the lane change is performed in anticipation of an improvement in the immediate driving conditions. On the other hand, an MLC sub-manoeuvre is performed due to compulsion arising from traffic rules (e.g., stalled vehicle, need to follow desired route, etc.). Moreover, the lane change to return back to the original lane can also be either DLC or MLC based on traffic conditions in each lane, legislation, etc. thus, transforming an overtaking manoeuvre into a complex task of dynamically choosing the best driving lane based on (i) legislation, (ii) driving intentions, and (iii) instantaneous traffic situation. This inference that the choice of lane is affected by both; (i) driving intention, and (ii) neighbourhood traffic conditions was verified in [7] using an integrated model (combining MLC and DLC) for lane changing behaviour based on gap acceptance (lead and lag gap). Therefore, it is noted that due to the dynamic nature of driving environments (i.e., traffic conditions in original and fast lane, speed limits, road conditions, etc.) overtaking is not standardised manoeuvre and thus, each overtaking manoeuvre in real-world scenarios is unique. This uniqueness arises from variations in number of overtaken vehicles, dura40 tion of overtake, relative velocity between concerned vehicles, distance between concerned vehicles, etc. [8 15]. For an autonomous vehicle, feasibility of an overtaking manoeuvre is evaluated on the basis of safety based on subject vehicle's states as well as surrounding information leading to a discrete outcome for making tactical decisions (i.e., either perform lane-change or do not perform lane 45 change) which form a part of planning and decision making process. A variety of techniques for decision making are available in literature with (i) multi-level decision trees [16], (ii) probabilistic weighted comparison of concurrent goals [17, and (iii) higher award seeking Markovian Decision Process algorithms [18] being among the prominent methods.

A schematic representation of an overtaking manoeuvre is shown in Figure 1 with each sub-manoeuvre labelled with roman numerals. As discussed 
above, the lane change back to the original lane depends on the traffic conditions and thus both possibilities are are depicted in the schematic. Despite the innumerable variations present due to the factors discussed above, overtaking manoeuvres can be classified under the four categories listed below [10]:

- Normal: The subject vehicle approaches the lead vehicle and waits for a suitable opportunity to perform the manoeuvre

- Flying: The subject vehicle does not adjust its longitudinal velocity and is directly able to overtake the lead vehicle

- Piggy backing: The subject vehicle follows a preceding vehicle as they both overtake the lead vehicle

- $2^{+}$: The subject vehicle overtakes two or more lead vehicles in a single manoeuvre

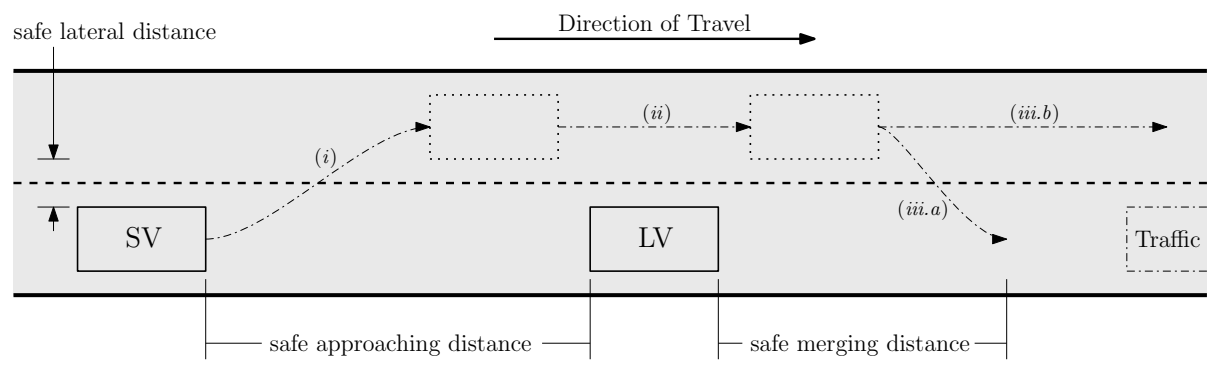

Figure 1: Basic schematic of an overtaking manoeuvre. Note: Different sub-manoeuvres are (i) lane-change; (ii) pass lead vehicle; (iii.a) merge back into original lane; (iii.b) continue in faster lane to pass traffic

For the aforementioned scenarios, the duration of a completed overtake has been found to be in the range of 5.4 to 12.5 seconds (subject to dynamic nature of the surrounding traffic and environment) using recording the trajectories of vehicles on typical European highways [3, 14, 19, 23]. Performing an autonomous overtaking manoeuvre based on any of scenarios mentioned above within a given time range requires accurate information of surrounding environment, traffic, and weather conditions along with sophisticated sensing and 
perception, planning, and control systems 24]. The surrounding environment of a vehicle is populated by different features; (i) permanent (road and lane limits), (ii) slowly changing (e.g., temporary speed limits, road works, traffic density, etc.), and (iii) fast changing (surrounding vehicle velocity, position, 75 heading, etc.). A modern day vehicle uses a host of on-board sensors to discern the environment and the placement of an on-board sensor suite used to perform this task can be seen in Figure 2. The information from these sensors is combined and used for tasks such as; (i) classify objects, (ii) track stationary and moving obstacles, (iii) identify safe driving zones, etc. Currently, there are some production vehicles that utilise vehicle-to-everything (V2X) information to provide updates on permanent (e.g., road and lane limits, road inclination, etc.) or slowly changing features (e.g., temporary speed limits, road works, traffic updates, etc.) of surrounding environment via a combination of cellular data and Local Dynamic Map (LDM) updates. However, despite an elaborate sensor suite and first generation V2X communication systems the capabilities of the contemporary autonomous vehicles is limited to low-speed overtaking. This is due to limitations such as; (i) range of sensors, (ii) blind spots , (iii) small time-scales for predicting motion of traffic participants, (iv) sensor imperfections, and ( $\mathrm{v}$ ) possible V2X network outages. The combination of one 90 or more of these limitations result in significant uncertainty while planning complex highway manoeuvres (e.g., overtaking) which span several seconds at high-speeds 25, 26. Moreover, unless all the traffic participants are connected and autonomous the uncertainty arising from predicting the motion of traffic vehicles cannot be brought down to negligible levels even with the advent of perfect on-board sensors and/or V2X communication network. Thus, predicting the motion of traffic participants for risk assessment forms a vital part of manoeuvre planning and this domain has witnessed a lot of research and a large number of techniques are present in literature. The different methods for motion planning for intelligent autonomous vehicles based on abstraction levels of traffic motion are classified as; (i) Physics-based 27-29], (ii) Manoeuvre-based [30, and (iii) Interaction-aware [31, 32]. A comprehensive survey discussing the 
advantages and limitations of each of these techniques is presented in [33] and an interested reader is directed towards it.

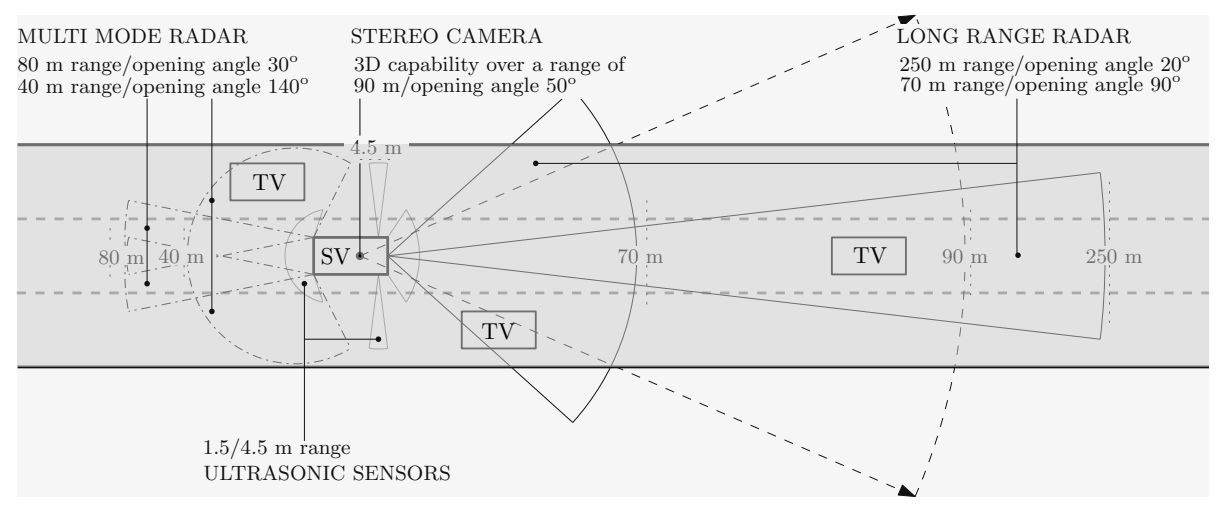

Figure 2: Visibility of an autonomous vehicle. Note: SV: Subject Vehicle, TV: Traffic Vehicle. Sensor performance specifications are based on 34]

Recent research has highlighted the potential use of off-board information

via V2X communications in expanding the sensory and perception horizon of a vehicle through the communication systems 3537. In the context of autonomous overtaking, initial research has been largely focused on the integration of V2X information to: (i) manoeuvre feasibility check, and (ii) decision making stages [9, 10, 35]. However, the potential enhancements that can be achieved in trajectory planning and trajectory tracking of an overtaking manoeuvre by exploiting V2X information are yet to be studied. In this paper, a review of various techniques for trajectory planning and trajectory tracking for autonomous overtaking systems is presented. The aim of this paper is twofold: (i) to gain insight on techniques suitable for autonomous overtaking systems, and (ii) to investigate how $\mathrm{V} 2 \mathrm{X}$ information can enhance both trajectory planning and tracking techniques of an autonomous overtaking system.

The paper is structured as follows: Section 2 introduces the system overview of an autonomous driving system and discusses how a 2-tier control architecture can be used to perform autonomous overtaking. In Section 3, an extensive literature review of trajectory planning methods used for generating overtaking 
trajectories is presented. Comparison of key aspects pertaining to vehicle models and a review of different control strategies for trajectory tracking applications is performed in Section 4. Finally, the concluding remarks are presented in Section 5

\section{System Architecture}

An autonomous overtaking manoeuvre requires consideration of a variety of factors such as subject vehicle states and constraints, lead vehicle states, environment limits, safety, and comfort. An overview of an intelligent autonomous driving system capable of performing autonomous overtaking is shown in Figure 3 For an autonomous vehicle to successfully perform different tasks (e.g., lane change, pass lead vehicle, and merge) pertaining to overtaking, it is expected that the vehicle can carry out each sub-task within the sensing and perception, planning, and control blocks. Sensing and perception includes gathering information about the driving conditions to determine if and when the tation 38 .

The planning module utilises the perception information along with the subject vehicle states and dynamic constraints to compute safe collision free local trajectory for the subject vehicle at each time instant [39]. To plan an overtaking manoeuvre the vehicle uses perception data (position and velocity estimates of neighbouring vehicles, infrastructure limits, road geometry, headway time) and subject vehicle data (current state, lateral and longitudinal dynamics) to check feasibility of the manoeuvre and design a collision free and safe local reference trajectory for an overtaking manoeuvre 3 , 15, 40,44]. 




Figure 3: Overview of an autonomous driving system (e.g., $X_{\text {ref }}, Y_{\text {ref }}$, and $v_{\text {ref }}$ ) for the vehicle to track [42]. The trajectory tracking 
controller then computes, via feedback algorithms based on the tracking error, the necessary torque $\left(\tau_{\text {ref }}\right)$ and steering inputs $\left(\delta_{\text {ref }}\right)$ required to track the reference, despite possible measurement noise, un-modelled dynamics, parametric uncertainties which may or may not be accounted for by the trajectory planning controller.

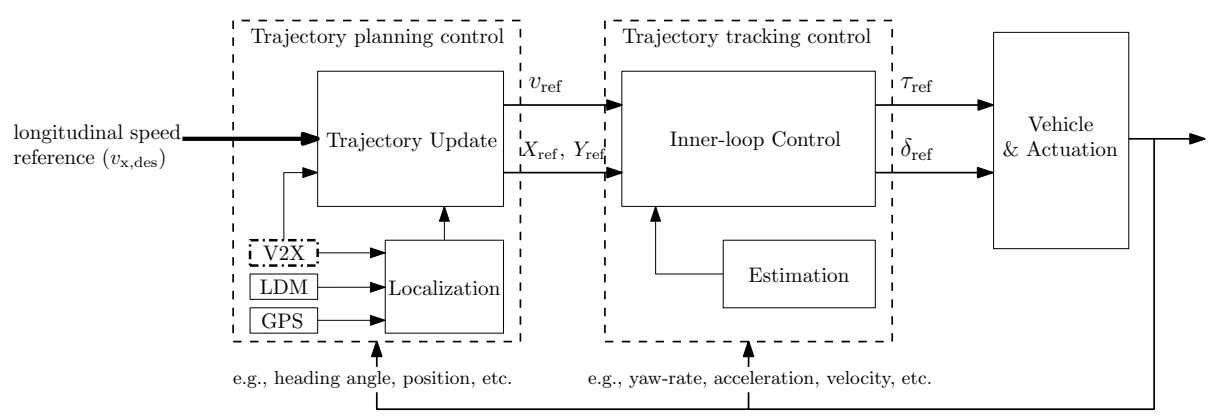

Figure 4: General control architecture for an autonomous vehicle [38, 43, 49, 51]. (V2X block with dot-dash boundary: optional functionality)

\section{Trajectory Planning}

An autonomous vehicle relies on real-time vehicle state and environment information (e.g., surrounding vehicles, road conditions) to derive a local trajectory that ensures a safe passage while minimising the deviation from the overall journey trajectory (global trajectory). Local trajectory planning can be defined as - real-time planning of the vehicle's transition from one feasible state to the next while satisfying the vehicle's kinematic limits based on vehicle dynamics and constrained by occupant comfort, lane boundaries and traffic rules, while, at the same time, avoiding obstacles [39. Technical literature shows that the vast majority of trajectory planning methods for an overtaking application employ one of the four well known techniques i.e., potential fields, cell decomposition, interdisciplinary methods and optimal control. In this section, these techniques are reviewed to gain insight into their performance for different specifications such as computational requirements, safety, feasibility in high-speed overtaking and real-time implementation. 
Potential field algorithms assign repulsive fields to obstacles and attractive fields to safe zones of the vehicle and then use an algorithm to compute trajectories along the steepest potential gradient in the resulting field 42 , 43, see Figure 5a. The computed path is guaranteed to follow the lowest potential (i.e., find collision free trajectory) in a given space but its safety and accuracy depends heavily on the accuracy of the generated potential field (i.e., definite knowledge of position of stationary and moving obstacles). However, due to the high computation costs and need for very accurate surrounding environment information, the method has only been experimentally verified for low speed (i.e., urban) manoeuvres 43. Additionally, it is seen that the algorithm cannot handle vehicle kinematic constraints which may cause safety issues in high-speed driving scenarios [42, 52].

Cell decomposition algorithms such as Rapidly-exploring Random Tree (RRT) is a method used for collision free path planning [53, 54, see Figure 5b. These algorithms can be modified to incorporate the vehicle constraints but they also suffer from computational and memory costs $42,53,54$. The computational complexity of such algorithms increases with increasing traffic density and frequency of road curvature thus jeopardizing the on-board computation of an autonomous vehicle on busy roads [53. Furthermore, the paths created by RRT's are jerky and tracking such a trajectory will have an adverse effect on the comfort of the occupants [39].

Inter-disciplinary techniques inspired by robotics and missile guidance systems [5, 55, 56] for vehicle path-planning are also reported in literature. One of the novel approaches proposed was to use motion primitives (combination of steady-state equilibrium trajectories and pre-specified manoeuvres) [57]. The experimental results demonstrated that collision free and feasible trajectories can be generated in real-time using this approach [57. Ghumman et al. designed a trajectory planning method based on Rendezvous Guidance technique (passing vehicle is guided in real-time to match the position and velocity of a shadow target during an overtaking manoeuvre) inspired from missile guidance systems [55, 56], see Figure 5c. Similarly, an approach for overtaking manoeu- 
vre consisting of consecutive tracking of virtual reference points positioned a priori at known distances from the lead vehicle is proposed in [5]. Simulation results of both these approaches demonstrated acceptable real-time capabilities for generating feasible trajectories but tracking performance was validated using low order models in computer simulations. Thus, in the absence of experimental validation it is difficult to form conclusions on the efficacy of such approaches.

Optimal control methods minimise a performance index (e.g., change in kinetic energy [15], jerk [24, 52, lateral acceleration [52]) under a set of constraints (e.g., vehicle lateral and longitudinal limits, environment constraints, neighbouring vehicles) to obtain a trajectory for a safe overtaking manoeuvre. The results from literature demonstrate that the method is successful in generating collision free trajectories without high computational requirements [15, 24, 52]. The autonomous vehicle JUNIOR developed by Stanford University has successfully demonstrated the effectiveness of optimal control based trajectory planning techniques at the DARPA Urban Challenge [58]. In this control framework, the researchers design two sets of trajectories, one for lateral motion and another for longitudinal motion each optimised for safety and occupant comfort. A set of combined lateral and longitudinal motion is obtained by combining these two sets. The final trajectory that is provided to the trajectory tracking controller is computed by following the steps; (i) filter out trajectories that breach safety and comfort limits, (ii) use filtered set of trajectories to identify ideal trajectory that minimises deviation from the road centre. However, most of these techniques do not take into account the non-linearities in the vehicle and tire dynamics resulting in unfeasible trajectories under high-speeds and/or low road friction conditions which pose a safety risk for autonomous vehicles [50]. Additionally, trajectories obtained by such open-loop single stage optimisation do not account for uncertainties in a dynamic environment and therefore these trajectory planning methods have limited potential unless used in either extremely controlled or structured environments.

Recently, Model Predictive Control (MPC) methodology has also been used by researchers for local trajectory planning, due to its ability to better handle 
system constraints and nonlinearities, see Figure $5 \mathrm{~d}$. The approach involves solving a constrained finite-time optimal control problem to determine a sequence of control inputs that minimise a performance index (cost function) and applying the optimal inputs (e.g., steering wheel angle, throttle, and brake) using a receding horizon principle [47. However, the presence of (i) nonlinear vehicle dynamics, and (ii) time-varying state and input constraints while navigating in a dynamic environment, leads to a nontrivial control problem thus presenting a computational burden to solve the optimisation problem in realtime [4]. Researchers have attempted to reduce the computational complexity arising due to the nonlinear vehicle dynamics by using (i) point mass vehicle model [38, 46, 51, (ii) linear kinematic bicycle vehicle model [45, 48, 50] and (iii) iterative linearisation of nonlinear vehicle model [47, in the prediction model. It is noted that the collision avoidance constraints are non-convex in nature which means that the feasibility and uniqueness of the optimisation cannot be guaranteed. Researchers have proposed different techniques (translating problem from time-dependent system to position-dependent system [38, 46, 50, 59, relaxing collision avoidance constraints [51, approximate linearisation [47] to guarantee uniqueness of solution and reduce the computing and memory requirements of the controller. The experimental results demonstrate the ability of these approaches to generate safe collision free trajectories around static or moving obstacles (i.e. overtaking manoeuvre) but it should be noted that these path-planner methods required exact knowledge of the states, of the obstacles (stationary, moving) and/or a high performance computing platform (desktop class computer) to calculate safe collision free trajectories [38, 45, 48, 50, 51]. It is noteworthy that recent publications have demonstrated that computing constraints may soon become an issue of the past as highly efficient algorithms for implementing MPC controllers on real-time prototyping systems and vehicle electronic control units have been developed and a few successful implementations are discussed in 60 62. Among the reviewed approaches, MPC provides a promising approach for trajectory planning due to its ability to: (i) include sys275 tem dynamics and constraints, and (ii) perform receding horizon control which 


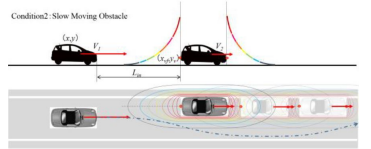

(a)

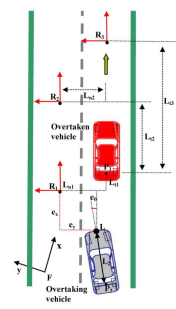

(c)

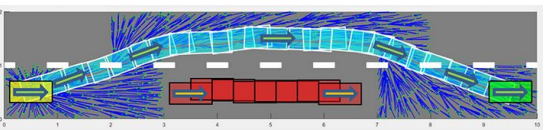

(b)



(d)

Figure 5: Trajectory planning via (a) Potential Fields [43]; (b) RRT 63]; (c) Virtual Reference Tracking [5]; (d) Model Predictive Control [4]

allows it to plan feasible trajectories over a larger operating range.

It is noteworthy that all methods discussed above operate under the assumption that accurate knowledge of the environment and lead vehicle states are available on-demand to the trajectory planning system. The advantages propose different ways for dealing with the uncertainty in current environment 
perception and limited future prediction capabilities. Potential field and cell decomposition based methods assign additional buffer zones (based on headway time, instantaneous relative velocity, etc.) around each obstacle and thus the search for feasible trajectories is performed in a constrained search space [64. Similarly, the trajectory planning techniques in [5, 55, 56] also compute virtual target points conservatively by expanding the margins of the virtual reference points in accordance with the relative velocities of the subject and lead vehicle. On the other hand, a type of MPC control technique known as Scenario-Based MPC (SCMPC) has been proposed in literature to mitigate the uncertainty arising due to traffic interactions in a systematic manner [45, 60, 65, 66. In this approach either an interaction-aware traffic prediction model [45] or manoeuvre based traffic prediction model [60] is incorporated within the MPC framework to simulate traffic scenarios as a probability distribution and a finite horizon optimal control problem is solved to generate a trajectory that is safe, feasible, and admissible under a selected set of traffic scenarios. The efficacy of the SCMPC trajectory planning technique for generating safe lane change manoevures has been demonstrated numerically and its real-time capability has been experimentally validated $45,60,65,66$. However, the effectiveness of this method has a dependence on the accuracy of the modelled traffic scenarios which makes obtaining large quantity of actual traffic data a necessity. Recently, it has been proposed by researchers that a V2X communication system can augment a vehicle's sensing and perception capabilities to potentially mitigate the issues discussed above 9 , 10, 35, 45, 67, 68. Initial studies for trajectory planning using the information obtained through V2X systems, suggest that the safety and feasibility of a manoeuvre can be enhanced by incorporating off-board information 69 71. Nonetheless, tangible benefits of using off-board information (e.g., lead vehicle states, road conditions, etc.) in trajectory planning methods are not very clearly understood and thus such studies are open to further research. Nonetheless, how a V2X system capable of providing accurate surrounding (e.g., lead vehicle states, road conditions, etc.) information in real-time can improve trajectory planning methods needs to be understood and is a question open to 
further research. Moreover, a wireless information sharing system induces additional dynamics related to communication delays, packet losses, and connection drop-outs which adds to the complexity of a control system 72 . Therefore, meticulous studies are required to ensure that the trajectory planning methods are robust and fault-tolerant against such network imperfections [73].

\begin{tabular}{|c|c|c|}
\hline Control Strategy & Strength(s) & Weakness \\
\hline Potential fields & $\begin{array}{l}\text { - Optimality of searched path } \\
\text { guaranteed } \\
\text { - Collision free path guaranteed }\end{array}$ & $\begin{array}{l}\text { - High computation cost } \\
\text { - Inability to handle system con- } \\
\text { straints } \\
\text { - No systematic procedure to } \\
\text { consider environmental uncer- } \\
\text { tainties }\end{array}$ \\
\hline $\begin{array}{l}\text { Cell Decomposi- } \\
\text { tion }\end{array}$ & $\begin{array}{l}\text { - Guaranteed collision free tra- } \\
\text { jectories }\end{array}$ & $\begin{array}{l}\text { - Computation requirements } \\
\text { sensitive to traffic density } \\
\text { - Computed paths are jerky } \\
\text { - No systematic procedure to } \\
\text { consider environmental uncer- } \\
\text { tainties }\end{array}$ \\
\hline $\begin{array}{l}\text { Interdisciplinary } \\
\text { Techniques }\end{array}$ & $\begin{array}{l}\text { - Reduced complexity of collision } \\
\text { avoidance as trajectory planning } \\
\text { converted to reference tracking } \\
\text { problem } \\
\text { - Real-time capable }\end{array}$ & $\begin{array}{l}\text { - Experimentally unproven } \\
\text { - No systematic design proce- } \\
\text { dure } \\
\text { - Do not consider uncertainties } \\
\text { in environment perception while } \\
\text { generating reference points }\end{array}$ \\
\hline Optimal Control & $\begin{array}{l}\text { - Generate collision free trajec- } \\
\text { tories } \\
\text { - Ability to include kinematic } \\
\text { constraints }\end{array}$ & $\begin{array}{l}\text { - Unsuitable for high-speed driv- } \\
\text { ing manoeuvres with large angles } \\
\text { of tire slip } \\
\text { - Inability to consider tire dy- } \\
\text { namics }\end{array}$ \\
\hline $\begin{array}{l}\text { Model Predictive } \\
\text { Control (MPC) }\end{array}$ & $\begin{array}{l}\text { - Include vehicle and tire dynam- } \\
\text { ics } \\
\text { - Systematic handling of con- } \\
\text { straints and traffic uncertainties } \\
\text { - Computational requirements } \\
\text { independent of environment }\end{array}$ & $\begin{array}{l}\text { - Optimisation sensitive to num- } \\
\text { ber of constraints } \\
\text { - Computation complexity scales } \\
\text { quickly with high-order system } \\
\text { models, non-linearity, and non- } \\
\text { convexity of constraints }\end{array}$ \\
\hline
\end{tabular}

Table 1: Summary of techniques for trajectory planning to avoid a moving obstacle 


\section{Trajectory Tracking}

330 field with a plethora of control methodologies available in literature dating all the way back to the middle of the $20^{\text {th }}$ century. Some useful properties for assessing tracking controllers for autonomous vehicle applications are listed below [74.

- Real-time capability: The control law needs to be implementable on a ve-

hicle's Electronic Control Unit (ECU) and function within the calculation time

- Robustness: The designed controller should be robust against system nonlinearities, model parameter variations, and external disturbances

- Operating Range: The tracking controller should ideally work across the entire range of vehicle speeds $(0-120 \mathrm{~km} / \mathrm{h})$

- Controller parameter tuning: A systematic tuning procedure for the controller parameters allows for a structured controller design procedure

The performance of closed-loop tracking controllers depends on the accuracy of the modelled system dynamics. Vehicle models used for capturing the dynamics should provide a trade-off between model accuracy and fidelity. In literature a variety of vehicle models (ranging from low dimension point mass-models to high-fidelity multi-body models) are presented. Different vehicle models that have been developed over the years to capture the longitudinal, lateral and yaw dynamics of a vehicle have been documented in [75]. Out of the wide variety of vehicle models available in literature a kinematic bicycle model and dynamic bicycle model have been found to provide a good compromise between model complexity and accuracy for controller design related to highway driving applications [61, 76]. A comprehensive review of trajectory tracking control on the aspects of choice of vehicle model, control strategies, and controller performance criteria has been performed in [77. The review demonstrated that geometric models based on Ackermann steering are not suitable for high-speed trajectory 
tracking due to their inability to include vehicle dynamics (e.g., acceleration and velocity). Additionally, it is highlighted that kinematic models (bicycle, four-wheel) are also unsuitable for high-speed trajectory tracking as they are inaccurate in regions of tire force saturation. Both linear and non-linear dynamic vehicle models (full vehicle model, half vehicle model, and bicycle model) were found to mitigate these limitations and furthermore providing a more accurate representation of a vehicle during high-speed driving [77. However, it was also shown that a dynamic bicycle model (linear) was suitable for driving tasks (lane-change manoeuvre, overtaking manoeuvre, highway driving) with small lateral acceleration $(\leq 0.5 \mathrm{~g})$ and low vehicle side-slip angle $\left(5^{\circ}\right)$ [77, 78. Most of the papers in literature have used a single-track vehicle model (bicycle model) for developing a tracking controller for performing overtaking manoeuvres since an overtaking manoeuvre is performed well within the dynamic limits of the vehicle (i.e., lateral acceleration, vehicle side-slip, and yaw-rate) where both the vehicle as well as tire dynamics can be approximated by linear models. However, at high-speeds and/or under low road friction overtaking scenarios, it is quite possible that the system (i.e., vehicle, and tires) may exhibit significant non-linear behaviour and therefore for appropriate scenarios either nonlinear models, linear parameter varying (LPV) models or multiple models can be used to capture the relevant dynamic behaviour of the system [78, 79]. For a detailed review of different vehicle models the reader is directed towards the work by [77, 80, 82.

\subsection{Tracking Controllers}

A comparison of different tracking controllers for autonomous vehicles was performed in $[77,80,82$. Some relevant observations of these comparisons along with other examples of tracking controllers for autonomous overtaking are discussed below.

Geometric controllers are designed using geometric vehicle models [77, 80-

82. Pure-pursuit and Stanley method are two prevalent geometric controllers [77, 80, 82. Pure-pursuit is a technique where the vehicle is in constant pursuit 
of a virtual moving point in front of the vehicle and 'Stanley' controller is based on non-linear geometric controller which considers heading and lateral error to compute steering angle corrections 77. These type of controllers (pure pursuit, Stanley, etc.) are easy to implement but are suitable only for applications that do not need to consider vehicle dynamics. Furthermore, since this approach does not follow a systematic control parameter tuning method, it is difficult to achieve a trade-off between stability and tracking performance 80 82. It is observed that over-tuning of both pure-pursuit and Stanley controllers leads to poor tracking performance during cornering [80]. Kinematic controllers are alternative control techniques for trajectory tracking. They are feedback controllers which are designed considering the vehicle kinematics (e.g., longitudinal velocity, lateral velocity, yaw-rate, etc.). Kinematic controllers have been shown to improve the tracking performance provided by geometric controllers but the gains over a geometric controllers are not high enough to justify the additional effort involved in designing and tuning the controller [77, 80, 81. Moreover, since these methods ignore vehicle dynamics, their applicability in critical driving environments (e.g., high-speed driving, extreme path curvature, etc.) cannot be assured.

Examples of classical control algorithms (e.g., PID, sliding mode controller) are also found in literature. Tracking controllers using classical techniques (PID) are shown to have good tracking performance but tuning of the parameters was found to be major challenge due to the presence of vehicle and tire nonlinearities. Sliding Mode Control (SMC), a well-established classical non-linear state-feedback controller has also been used to design vehicle trajectory tracking controllers and shows good tracking accuracy due to the non-linear control law [77, 83. However, it suffers from a few drawbacks namely: (i) performance is sensitive to the sampling rate of the controller (ii) chattering problems, (iii) robustness only on the sliding surface, and (iv) needs prior knowledge of disturbance and uncertainty bounds [77, 82, 83].

Dynamic state feedback (linear and nonlinear) based control methods demonstrate better performance that geometric and kinematic controllers as they con- 
sider the dynamics of the vehicle and tires while computing the control law. Linear Quadratic Regulator (LQR) based control law is easy to design but while tracking trajectories with varying curvature feedforward control is required to achieve error-free tracking. However, adding feedforward control makes the tracking controller sensitive to discontinuities in the reference trajectory which requires additional tuning to attenuate $[80$. On the other hand, optimal control based methods can provide accurate trajectory tracking even at high-speeds but this is achieved only when certain assumptions (e.g., velocity of the subject vehicle remains constant during the optimisation horizon) are fulfilled. Recently, nonlinear adaptive control techniques such as Inversion \& Immersion (I\&I) have also been used for vehicle trajectory tracking controllers. Initial studies demonstrate that this method provides robust closed-loop tracking performance but the controller is sensitive to parameter uncertainties 83. In the same body of work, an adaptive Proportional-Integral (PI) with non-linear gains controller for trajectory tracking was also proposed. 83. Simulation results indicate that the controller provides tracking performance at par with an SMC and I\&I controller with added advantage in the form of insensitivity to parameter uncertainties. However, in presence of large curvature variations or when operated in non-linear region of vehicle dynamics, the controller gains have a tendency to become high which may have a detrimental effect on the actuators.

There are also examples of advanced model based control techniques such as MPC being used for vehicle trajectory tracking [38, 46, 48, 50, 51, 57,. Nonlinear MPC was found to provide very accurate tracking performance but at the same time suffer due to computational requirements of online optimisation 84]. To reduce the computational burden researchers use a linear vehicle model but such controllers are applicable only in linear region of vehicle and tire behaviour 45, 48]. Designing a MPC framework based on iterative linearisation of a non445 linear model has been proposed as a way to expand the working range of linear MPC controllers for trajectory tracking and has been experimentally validated [47. This approach helps in meeting the compromise between computational requirements and modelling errors. 
Neural network and fuzzy logic based approaches have also been proposed prevent performance drop-off. However, the order of a controller rises with the number robustness criteria that are incorporated and the number of controllers in a 'bank' scales exponentially with the number of varying parameters making 
to be limited to certain pre-set conditions and scenarios. However, the range of benefits (e.g., tracking performance, safety improvements, etc.) that can be gained by such a system needs further investigation resulting in an open research question.

\begin{tabular}{|c|c|c|}
\hline Control Strategy & Strength(s) & Weakness \\
\hline $\begin{array}{l}\text { Geomteric \& Kine- } \\
\text { matic }\end{array}$ & $\begin{array}{l}\text { - Adequate performance (experi- } \\
\text { mentally validated) in conditions } \\
\text { without disturbances (e.g., wind, } \\
\text { road banking) } \\
\text { - Good tracking performance } \\
\text { and robustness at moderate } \\
\text { speeds (e.g., kinematic) }\end{array}$ & $\begin{array}{l}\text { - Do not consider vehicle dynam- } \\
\text { ics } \\
\text { - Steady-state error increases for } \\
\text { high-speed driving (e.g., geomet- } \\
\text { ric) } \\
\text { - Unsuitable for high-speed driv- } \\
\text { ing as dynamics are neglected } \\
\text { (e.g., kinematic) } \\
\text { - Requires smooth and continu- } \\
\text { ous reference trajectories }\end{array}$ \\
\hline
\end{tabular}




\begin{tabular}{|c|c|c|}
\hline Classical & $\begin{array}{l}\text { - Established method with good } \\
\text { performance for non-linear sys- } \\
\text { tems } \\
\text { - Robust closed-loop perfor- } \\
\text { mance against uncertainties and } \\
\text { noise (e.g., SMC) }\end{array}$ & $\begin{array}{l}\text { - Tuning of controller parame- } \\
\text { ters is tricky (e.g., PID) } \\
\text { - Robust performance only in } \\
\text { limited scenarios (e.g., SMC) } \\
\text { - Control law is sensitive to path } \\
\text { curvature variations (e.g., SMC) }\end{array}$ \\
\hline $\begin{array}{l}\text { Dynamic state } \\
\text { feedback }\end{array}$ & $\begin{array}{l}\text { - Consider vehicle dynamics in } \\
\text { calculating control law } \\
\text { - Optimisation shifted offline re- } \\
\text { sulting in simple implementation } \\
\text { of control law }\end{array}$ & $\begin{array}{l}\text { - Obtaining vehicle states (e.g., } \\
\text { wheel forces, slip angles, torques } \\
\text { etc.) is non-trivial } \\
\text { - Control law is sensitive to path } \\
\text { curvature variations (e.g., LQR) }\end{array}$ \\
\hline Neural Network & $\begin{array}{l}\text { - Sufficient training can make } \\
\text { the behaviour very human-like to } \\
\text { make the automated car feel nat- } \\
\text { ural }\end{array}$ & $\begin{array}{l}\text { - Controller tuning requires sim- } \\
\text { ulation with large amounts of } \\
\text { real world (training) data } \\
\text { - No failure explanations possi- } \\
\text { ble }\end{array}$ \\
\hline Fuzzy Logic & $\begin{array}{l}\text { - Closed-loop system acts simi- } \\
\text { lar to a human-driver (because of } \\
\text { human-like rules) }\end{array}$ & $\begin{array}{l}\text { - Controller tuning is not sys- } \\
\text { tematic with no formal stability } \\
\text { analysis } \\
\text { - Rules can become unmanage- } \\
\text { able if number of variables is } \\
\text { large }\end{array}$ \\
\hline $\begin{array}{l}\text { Model Predictive } \\
\text { Control (MPC) }\end{array}$ & $\begin{array}{l}\text { - Systematic design procedure } \\
\text { - Ability to include system and } \\
\text { actuator constraints in design } \\
\text { procedure } \\
\text { - Inclusion of vehicle and tire dy- } \\
\text { namics in control problem }\end{array}$ & $\begin{array}{l}\text { - Non-linear MPCs with have } \\
\text { high computing requirements } \\
\text { making them unsuitable for } \\
\text { high-speed driving environments } \\
\text { - The tracking performance is } \\
\text { sensitive to the accuracy of pre- } \\
\text { diction model } \\
\text { - Larger tuning parameter set } \\
\text { compared to industry standard } \\
\text { PID }\end{array}$ \\
\hline
\end{tabular}

Table 2: Summary of control strategies for vehicle trajectory tracking $[74,77,80,81,83$ 
This paper reviewed different approaches towards trajectory planning tracking for autonomous overtaking. The review of trajectory planning methods brings forth the following important aspects. First, vehicle dynamics, constraints and surrounding environment information needs to be considered while designing a trajectory for an overtaking manoeuvre and methods that incorporate these requirements within their framework are suitable candidates for real-world applications. Second, the trajectory planning techniques depend on accurate surrounding environment information, and off-board information via V2X communication can aid in expanding the accuracy and perception horizon thereby reducing safety concerns that might arise due to diverse driving conditions. For tracking controllers, the review showed that: (i) control algorithms that considered vehicle and tire dynamics over large speed ranges provided accurate tracking even at high-speeds and/or large trajectory variations, and (ii) the effectiveness of such controllers hinges on the accuracy of the modelled system dynamics which has difficulty in capturing the large variations encountered typically in daily driving with one low order system. Examples from literature showed that off-board information via V2X systems can be used to update controller parameters in real-time which can prevent drop-off in tracking performance when operated in conditions with variations in system dynamics. However, integration of off-board information into a multi-tier control architecture needs to be seamless as well as capable of graceful degradation on occasions of wireless communication failure. This added complexity in control design can pose significant challenges that will need to be addressed to develop a safe, dependable, and robust control system.

It is noteworthy that the study of potential benefits that can be achieved by leveraging off-board information via V2X communication systems for autonomous trajectory planning and tracking is in a nascent stage and marks a new chapter of study in the field of autonomous vehicles. 


\section{Acknowledgement} EP/N01300X/1 as part of the jointly funded Towards Autonomy: Smart and Connected Control (TASCC) Programme.

\section{References}

[1] A. Eskandarian, Handbook of Intelligent Vehicles: Vol 2, 2012.

[2] T. Gordon, M. Lidberg, Automated driving and autonomous functions on road vehicles, Vehicle System Dynamics 53 (7) (2015) 958-994.

[3] V. Milanés, D. F. Llorca, J. Villagrá, J. Pérez, C. Fernández, I. Parra, C. González, M. A. Sotelo, Intelligent automatic overtaking system using vision for vehicle detection, Expert Systems with Applications 39 (3) (2012) 3362-3373

[4] B. Vanholme, D. Gruyer, B. Lusetti, S. Glaser, S. Mammar, Highly automated driving on highways based on legal safety, IEEE Transactions on Intelligent Transportation Systems 14 (1) (2013) 333-347.

[5] P. Petrov, F. Nashashibi, Modeling and Nonlinear Adaptive Control for Autonomous Vehicle Overtaking, IEEE Transactions on Intelligent Transportation Systems 15 (4) (2014) 1643-1656.

[6] S. Moridpour, G. Rose, M. Sarvi, Effect of surrounding traffic characteristics on lane changing behavior, Journal of Transportation Engineering 136 (11) (2010) 973-985.

[7] T. Toledo, H. Koutsopoulos, M. Ben-Akiva, Modeling Integrated LaneChanging Behavior, Transportation Research Record: Journal of the Transportation Research Board 1857 (03) (2003) 30-38.

[8] J. Baber, J. Kolodko, T. Noel, M. Parent, L. Vlacic, Cooperative autonomous driving: intelligent vehicles sharing city roads, IEEE Robotics \& Automation Magazine 12 (1) (2005) 44-49. 
[9] M. Motro, A. Chu, J. Choi, P. S. Lavieri, A. R. Pinjari, C. R. Bhat, J. Ghosh, R. W. Heath, Vehicular ad-hoc network simulations of overtaking maneuvers on two-lane rural highways, Transportation Research Part C: Emerging Technologies 72 (2016) 60-76.

[10] G. Hegeman, K. Brookhuis, S. Hoogendoorn, Opportunities of advanced driver assistance systems towards overtaking, EJTIR 5 (4) (2005) 281-296.

[11] C. Thiemann, M. Treiber, A. Kesting, Estimating Acceleration and LaneChanging Dynamics Based on NGSIM Trajectory Data, Transportation Research Record: Journal of the Transportation Research Board 2088 (2008) 90-101.

[12] A. Kesting, M. Treiber, D. Helbing, General Lane-Changing Model MOBIL for Car-Following Models, Transportation Research Record: Journal of Transportation Research Board 1999 (1) (2007) 86-94.

[13] N. A. Webster, T. Suzuki, E. Chung, M. Kuwahara, Tactical driver lane change model using forward search, in: Transportation Research Board 86th Annual Meeting, 2007, pp. 07-0378.

[14] E. I. Vlahogianni, Modeling duration of overtaking in two lane highways, Transportation Research Part F: Traffic Psychology and Behaviour 20 (2013) 135-146.

[15] T. Shamir, How Should an Autonomous Vehicle Overtake a Slower Moving Vehicle: Design and Analysis of an Optimal Trajectory, IEEE Transactions on Automatic Control 49 (4) (2004) 607-610.

[16] L. Claussmann, A. Carvalho, G. Schildbach, A path planner for autonomous driving on highways using a human mimicry approach with Binary Decision Diagrams, in: 2015 European Control Conference, ECC 2015, 2015, pp. 2976-2982. 
[17] M. Ardelt, C. Coester, N. Kaempchen, Highly automated driving on freeways in real traffic using a probabilistic framework, IEEE Transactions on Intelligent Transportation Systems 13 (4) (2012) 1576-1585.

[25] Y. S. Son, W. Kim, S. H. Lee, C. C. Chung, Robust multirate control scheme with predictive virtual lanes for lane-keeping system of autonomous highway driving, IEEE Transactions on Vehicular Technology 64 (8) (2015) 3378-3391. 
[26] M. Aeberhard, S. Rauch, M. Bahram, G. Tanzmeister, J. Thomas, Y. Pilat, F. Homm, W. Huber, N. Kaempchen, Experience, results and lessons learned from automated driving on Germany's highways, IEEE Intelligent Transportation Systems Magazine 7 (1) (2015) 42-57.

[27] R. Schubert, K. Schulze, G. Wanielik, Situation assessment for automatic

[31] A. Lawitzky, D. Althoff, C. F. Passenberg, G. Tanzmeister, D. Wollherr, M. Buss, Interactive scene prediction for automotive applications, in: 2013 IEEE Intelligent Vehicles Symposium (IV), 2013, pp. 1028-1033.

[32] M. Bahram, C. Hubmann, A. Lawitzky, M. Aeberhard, D. Wollherr, A com-

\section{Sensors, [Online; accessed 06-February-2018].}


URL http://www.caricos.com/cars/m/mercedes-benz/2018_ mercedes-benz_s-class/images/41.html

[35] Y. Luo, Y. Xiang, K. Cao, K. Li, A dynamic automated lane change maneuver based on vehicle-to-vehicle communication, Transportation Research Part C: Emerging Technologies 62 (2016) 87-102.

[36] S. Andrews, Vehicle-to-Vehicle (V2V) and Vehicle-to- Infrastructure (V2I) Communications and Cooperative Driving, in: Handbook of Intelligent Vehicles, 2012, pp. 1121-1144.

[37] L. Guzzella, Automobiles of the future and the role of automatic control in those systems, Annual Reviews in Control 33 (1) (2009) 1-10.

[38] B. Kim, D. Kim, S. Park, Y. Jung, K. Yi, Automated Complex Urban Driving based on Enhanced Environment Representation with GPS / map, Radar, Lidar and Vision, IFAC-PapersOnLine 49 (11) (2016) 190-195.

[39] C. Katrakazas, M. Quddus, W.-H. Chen, L. Deka, Real-time motion planning methods for autonomous on-road driving: State-of-the-art and future research directions, Transportation Research Part C: Emerging Technologies 60 (2015) 416-442.

[40] P. Saengpredeekorn, J. Srinonchat, A new technique to define the overtake distance using image processing, in: 2009 6th International Conference on Electrical Engineering/Electronics, Computer, Telecommunications and Information Technology, Vol. 02, 2009, pp. 1142-1145.

[41] R. Kala, K. Warwick, Motion planning of autonomous vehicles in a nonautonomous vehicle environment without speed lanes, Engineering Applications of Artificial Intelligence 26 (5-6) (2013) 1588-1601.

[42] S. Glaser, B. Vanholme, S. Mammar, D. Gruyer, L. Nouvelière, Maneuverbased trajectory planning for highly autonomous vehicles on real road with traffic and driver interaction, IEEE Transactions on Intelligent Transportation Systems 11 (3) (2010) 589-606. 
[43] S. Kitazawa, Control Target Algorithm for Direction Control of Autonomous Vehicles in Consideration of Mutual Accordance in Mixed Traffic Conditions, in: International Symposium on Advanced Vehicle Control $2016,2016$.

[44] A. Carvalho, S. Lefévre, G. Schildbach, J. Kong, F. Borrelli, Automated

[46] N. Murgovski, J. Sjöberg, Predictive cruise control with autonomous overtaking, in: IEEE 54th Annual Conference on Decision and Control (CDC), 2015, 2015, pp. 644-649.

[47] A. Carvalho, Y. Gao, A. Gray, H. E. Tseng, F. Borrelli, Predictive control of an autonomous ground vehicle using an iterative linearization approach, in: 16th International IEEE Conference on Intelligent Transportation Systems (ITSC 2013), no. ITSC, 2013, pp. 2335-2340.

[48] Y. Gao, A. Gray, H. E. Tseng, F. Borrelli, A tube-based robust nonlinear predictive control approach to semiautonomous ground vehicles, Vehicle System Dynamics 52 (6) (2014) 802-823.

[49] J. E. Naranjo, C. González, R. García, T. De Pedro, Lane-change fuzzy control in autonomous vehicles for the overtaking maneuver, IEEE Transactions on Intelligent Transportation Systems 9 (3) (2008) 438-450.

[50] Y. Gao, A. Gray, J. V. Frasch, T. Lin, E. Tseng, J. K. Hedrick, F. Borrelli, Spatial Predictive Control for Agile Semi-Autonomous Ground Vehicles, Proceedings of the 11th International Symposium on Advanced Vehicle Control VD11 (2) (2012) 1-6. 
[51] J. Nilsson, Y. Gao, A. Carvalho, F. Borrelli, Manoeuvre generation and control for automated highway driving, in: IFAC Proceedings Volumes (IFAC-PapersOnline), Vol. 19, IFAC, 2014, pp. 6301-6306.

[52] T. Shim, G. Adireddy, H. Yuan, Autonomous vehicle collision avoidance system using path planning and model-predictive-control-based active front steering and wheel torque control, Proceedings of the Institution of Mechanical Engineers, Part D: Journal of Automobile Engineering 226 (6) (2012) $767-778$.

[53] L. Ma, J. Xue, K. Kawabata, J. Zhu, C. Ma, N. Zheng, A fast RRT algorithm for motion planning of autonomous road vehicles, in: 2014 17th IEEE International Conference on Intelligent Transportation Systems, ITSC 2014, 2014, pp. 1033-1038.

[54] Y. Kuwata, G. A. Fiore, J. Teo, E. Frazzoli, J. P. How, Motion planning for urban driving using RRT, in: 2008 IEEE/RSJ International Conference on Intelligent Robots and Systems, IROS, 2008, pp. 1681-1686.

[55] G. Usman, F. Kunwar, Autonomous vehicle overtaking - An online solution, in: Proceedings of the 2009 IEEE International Conference on Automation and Logistics, ICAL 2009, no. August, 2009, pp. 596-601.

[56] U. Ghumman, F. Kunwar, B. Benhabib, Guidance-Based On-Line Motion Planning For Autonomous Highway Overtaking, International Journal On Smart Sensing And Intelligent Systems 1 (2) (2008) 549-571.

[57] A. Gray, Y. Gao, T. Lin, J. K. Hedrick, H. E. Tseng, F. Borrelli, Predictive Control for Agile Semi-Autonomous Ground Vehicles using Motion Primitives, in: 2012 American Control Conference (ACC), 2012, pp. 4239-4244.

[58] M. Werling, J. Ziegler, S. Kammel, S. Thrun, Optimal trajectory generation for dynamic street scenarios in a frenét frame, in: 2010 IEEE International Conference on Robotics and Automation (ICRA), 2010, pp. 987-993. 
[59] J. Karlsson, N. Murgovski, J. Sjöberg, Temporal vs. spatial formulation of autonomous overtaking algorithms, in: 2016 IEEE 19th International Conference on Intelligent Transportation Systems (ITSC), 2016, pp. 1029 1034.

[60] G. Cesari, G. Schildbach, A. Carvalho, F. Borrelli, Scenario Model Predictive Control for Lane Change Assistance and Autonomous Driving on $725 \quad$ Highways, IEEE Intelligent Transportation Systems Magazine 9 (3) (2017) 23-35.

[61] J. Kong, M. Pfeiffer, G. Schildbach, F. Borrelli, Kinematic and Dynamic Vehicle Models for Autonomous Driving Control Design, in: Intelligent Vehicles Symposium (IV), 2015 IEEE, 2015, pp. 1094-1099.

[62] G. Schildbach, E. F. Gmbh, A New Nonlinear Model Predictive Control Algorithm for Vehicle Path Tracking, in: International Symposium on Advanced Vehicle Control 2016, 2016.

[63] W. Khaksar, K. S. M. Sahari, T. S. Hong, Application of SamplingBased Motion Planning Algorithms in Autonomous Vehicle Navigation, Autonomous Vehicle.

[64] M. T. Wolf, J. W. Burdick, Artificial potential functions for highway driving with collision avoidance, in: Proceedings - IEEE International Conference on Robotics and Automation, 2008, pp. 3731-3736.

[65] G. Schildbach, L. Fagiano, C. Frei, M. Morari, The scenario approach for Stochastic Model Predictive Control with bounds on closed-loop constraint violations, Automatica 50 (12) (2014) 3009-3018.

[66] A. Carvalho, Y. Gao, S. Lefevre, F. Borrelli, Stochastic predictive control of autonomous vehicles in uncertain environments, in: Proc. 12th International Symposium on Advanced Vehicle Control, no. November 2016, 2014. 
[67] F. Damerow, B. Flade, J. Eggert, Extensions for the Foresighted Driver Model: Tactical lane change, overtaking and continuous lateral control, in: 2016 IEEE Intelligent Vehicles Symposium (IV), 2016, pp. 186-193.

[68] N. Ohara, M. Slot, J. Monteil, V. Cahill, M. Bouroche, Towards Evaluating the Benefits of Inter-vehicle Coordination, in: IEEE Conference on Intelligent Transportation Systems, Proceedings, ITSC, 2015, pp. 2444-2450.

[69] J. Pérez, V. Milanés, E. Onieva, J. Godoy, J. Alonso, Longitudinal fuzzy control for autonomous overtaking, in: 2011 IEEE International Conference on Mechatronics, ICM 2011 - Proceedings, 2011, pp. 188-193.

[70] F. You, R. Zhang, G. Lie, H. Wang, H. Wen, J. Xu, Trajectory planning and tracking control for autonomous lane change maneuver based on the cooperative vehicle infrastructure system, Expert Systems with Applications 42 (14) (2015) 5932-5946.

[71] Z. Huang, Q. Wu, J. Ma, S. Fan, An APF and MPC combined collaborative driving controller using vehicular communication technologies, Chaos, Solitons and Fractals 89 (2015) 232-242.

[72] H. Wymeersch, G. R. De Campos, P. Falcone, L. Svensson, E. G. Ström, Challenges for cooperative ITS: Improving road safety through the integration of wireless communications, control, and positioning, in: 2015 International Conference on Computing, Networking and Communications (ICNC), 2015, pp. 573-578.

[73] C. Houben, S. Houben, Endowing advanced driver assistance systems with fault tolerance, Annual Reviews in Control 39 (2015) 58-67.

[74] D. Watzenig, B. Brandstätter, Comprehensive Energy Management: Eco Routing \& Velocity Profiles, Springer, 2017.

[75] R. Rajamani, Vehicle Dynamics and Control, 2006. 
[76] C. M. Kang, S. H. Lee, C. C. Chung, Comparative evaluation of dynamic and kinematic vehicle models, in: 2014 IEEE 53rd Annual Conference on Decision and Control (CDC), 2014, pp. 648-653.

[77] N. Hafizah, A. Hairi, K. Hudha, Z. Abdul, Modelling and Control Strategies in Path Tracking Control for Autonomous Ground Vehicles : A Review of State of the Art and Challenges, Journal of Intelligent \& Robotic Systems.

[78] C.-i. Kim, M.-s. Kim, K.-s. Lee, Development of a Full Speed Range Pathfollowing System for the Autonomous Vehicle, in: International Conference on Control, Automation and Systems (ICCAS 2015), no. 15, 2015, pp. $710-$ 715.

[79] D. Filev, J. Lu, D. Hrovat, Future Mobility: Integrating Vehicle Control With Cloud Computing, Davor Mechanical Engineering 135 (3) (2013) 1824.

[80] J. M. Snider, Others, Automatic Steering Methods for Autonomous Automobile Path Tracking, Robotics Institute, Pittsburgh, PA, Tech. Rep. CMU-RITR-09-08.

[81] A. Sorniotti, P. Barber, S. D. Pinto, Path Tracking for Automated Driving: A Tutorial on Control System Formulations and Ongoing Research, in: Automated Driving: Safer and More Efficient Future Driving, 2017, pp. $71-140$.

[82] A. Rupp, M. Stolz, Survey on Control Schemes for Automated Driving on Highways, in: Automated Driving, 2017, pp. 43-69.

[83] G. Tagne, R. Talj, A. Charara, Design and Comparison of Robust Nonlinear Controllers for the Lateral Dynamics of Intelligent Vehicles, IEEE Transactions on Intelligent Transportation Systems 17 (3) (2016) 796-809.

[84] T. Besselmann, M. Morari, Autonomous vehicle steering using explicit LPV-MPC, 2009 European Control Conference (1) (2009) 2628-2633. 
[85] S. Chaib, M. S. Netto, S. Mammar, Hinf, Adaptive, PID and Fuzzy Control: a Comparison of Controllers for Vehicle Lane Keeping, in: IEEE Intelligent Vehicles Symposium, 2004, 2004, pp. 139-144.

[86] M. Jalalmaab, M. Pirani, B. Fidan, S. Jeon, Cooperative road condition estimation for an adaptive model predictive collision avoidance control strategy, IEEE Intelligent Vehicles Symposium, Proceedings 2016-Augus (Iv) (2016) 1072-1077. 